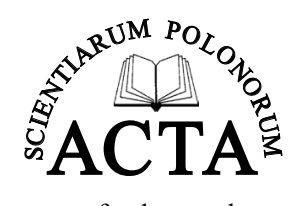

www.food.actapol.net

REVIEW PAPER
Acta Sci. Pol. Technol. Aliment. 18(2) 2019, 115-123

pISSN 1644-0730

eISSN 1898-9594

http://dx.doi.org/10.17306/J.AFS.2019.0654

Received: 26.03 .2019

Accepted: 16.05 .2019

\title{
POTENTIAL USE OF COLOSTRUM BOVINUM SUPPLEMENTATION IN ATHLETES - A REVIEW
}

\author{
Natalia Główka ${ }^{\bowtie}$, Małgorzata Woźniewicz \\ Institute of Human Nutrition and Dietetics, Poznań University of Life Sciences \\ Wojska Polskiego 31, 60-624 Poznań, Poland
}

\begin{abstract}
Colostrum Bovinum is the first milk secreted from the mammary glands of mammals after parturition. In calves, it is the only way to provide immunoglobulins, which are responsible for the proper maturation of the immune system. The benefits of calf colostrum have contributed to the investigation of the effects of colostrum in humans. Although the health properties of colostrum are not fully understood, its supplementation in athletes has been particularly interesting for over 20 years. The presence of growth factors, immunoglobulins, cytokines, lactoferrin and hormones suggests that colostrum may improve the functioning of the digestive, immune and neuroendocrine systems, and exercise performance. The administering of colostrum seems to be most effective during periods of high intensity training, probably due to its high concentration of IGF-I, the ability to increase muscle buffering capacity or its high SIgA concentration. Due to the lack of adjustment of specific doses and the period of colostrum supplementation, it is necessary to conduct further studies on the impact of colostrum supply on exercise performance. The aim of this review article is to analyze the available experimental studies and reviews on the use of colostrum in sport and to draw attention to the possibility of using colostrum to improve the health of athletes and the results they can achieve in their chosen sports.
\end{abstract}

Keywords: bovine colostrum, immunology, exercise performance, supplementation

\section{INTRODUCTION}

In recent years, bovine colostrum has received more attention in sports nutrition and exercise immunology, mainly due to the suggested beneficial immunological, health and recovery properties (Davison, 2012). The simultaneous presence of growth factors, immunoglobulins, cytokines, lactoferrin, lysozyme and growth hormone may improve the functioning of the immune and neuroendocrine systems and the integrity of the gastrointestinal tract, which may be impaired as a result of intense physical efforts (Shing et al., 2009).

The use of colostrum supplementation is increasingly popular and has been prevalent among athletes in recent times. Of particular interest is the potentially beneficial effect of colostrum and its bioactive supplies on the proper functionality of the immune, digestive and hormonal systems, and exercise performance (Shing et al., 2009).

\section{DEFINITION AND CHARACTERISTICS OF COLOSTRUM BOVINUM}

Colostrum, also known as bisnings, beestings or first milk, is a substance secreted from the mammary glands of mammals during the first few days after parturition 
Główka, N., Woźniewicz, M. (2019). Potential use of Colostrum Bovinum supplementation in athletes - A review. Acta Sci. Pol. Technol. Aliment., 18(2), 115-123. http://dx.doi.org/10.17306/J.AFS.2019.0654

Table 1. Bioactive ingredients in bovine colostrum (Bagwe et al., 2015)

\begin{tabular}{|c|c|c|}
\hline Nutrients & Immunological factors & Growth factors \\
\hline $\begin{array}{l}\text { Minerals (Ca, P, Mg, Na, K, Zn, } \\
\mathrm{Fe}, \mathrm{Cu}, \mathrm{S}, \mathrm{Mn} \text { ) } \\
\text { Vitamins (especially A, B12, E, } \\
\text { niacin, B1, B2) } \\
\text { Amino acids } \\
\text { Essential unsaturated fatty acids }\end{array}$ & $\begin{array}{l}\text { proline-rich polypeptides } \\
\text { immunoglobins (A, D, E, G, M) } \\
\text { lactoferrin } \\
\text { cytokine } \\
\text { lysozyme, oxidase, trypsin } \\
\text { leukocytes } \\
\text { T-, B-lymphocytes } \\
\text { oligopolysaccharides } \\
\text { orotic acid } \\
\text { others (e.g. sIgA, lactalbumin, beta-lactoglobulins) }\end{array}$ & $\begin{array}{l}\text { epithelial growth factor (EGF) } \\
\text { insulin-like growth factor (IGF-I, IGF-II) } \\
\text { fibroblast growth factor (FgF) } \\
\text { platelet-derived growth factor (PDGF) } \\
\text { transforming growth factor (TgA, TgB) } \\
\text { growth hormone }(\mathrm{GH})\end{array}$ \\
\hline
\end{tabular}

(24-72 h). Newborn babies only have access to the colostrum of their mothers during the first hours after birth, which forms the foundation for their long-term immunity (Bagwe et al., 2015). Human and bovine colostrum is a thick, sticky, jelly-like liquid containing many antibodies in a higher concentration than normal milk (Bagwe et al., 2015). Colostrum contains up to 40 times the number of biologically active compounds, 100-fold higher concentrations of SIgA, and 10-fold higher concentrations of lactoferrin than mature milk (Bagwe et al., 2015). The main growth factor in colostrum is insulin-like growth factor-I, which is important for stimulating the growth of muscle tissue and for maintaining muscle mass and its function (Francis et al., 1988). Colostrum is one of the strongest natural immune stimulants (Rak and Bronkowska, 2014). The ingredients contained within it can be divided into three main categories (Bagwe et al., 2015): nutritional components, immunological factors and growth factors (Table 1).

Comparing the composition of human and bovine colostrum (Table 2), it is worth noting the differences in protein, lactose and fat content (Bagwe et al., 2015).

Table 2. Comparison of human and bovine colostrum (Bagwe et al., 2015; Godhia and Patel, 2013)

\begin{tabular}{lcc}
\hline & Human colostrum & $\begin{array}{c}\text { Cattle colostrum } \\
\text { (cow) }\end{array}$ \\
\hline Energy, kcal/100 ml & 58 & 130 \\
Fat, \% & $3-5$ & 6.7 \\
Protein, \% & $0.8-0.9$ & 14.9 \\
Lactose, \% & $6.9-7.2$ & 2.5 \\
\hline
\end{tabular}

The caloric content of bovine colostrum is approximately $130 \mathrm{kcal}$ per $100 \mathrm{ml}$, whereas human colostrum has $58 \mathrm{kcal}$ per $100 \mathrm{ml}$ (Godhia and Patel, 2013). Exact values of the components of colostrum are presented in Table 3 (Bagwe et al., 2015; Godhia and Patel, 2013).

Newborns have a very small and immature digestive system. It has been shown that in their case the use of colostrum has a laxative effect, supports a healthy intestinal passage and helps to remove excess bilirubin to prevent jaundice. Antibodies contained in colostrum provide protection against infectious diseases, carrying out the required immunity response and the delivery of growth factors for the development of the digestive system (Bagwe et al., 2015).

Colostrum is considered to be a relatively safe substance for the human population. Nevertheless, the initial occurrence of side effects, such as nausea or bloating, is possible. People suffering from an allergy to cow's milk protein should not use colostrum (Bagwe et al., 2015).

Colostrum as a supplement should be produced organically and be free of additives such as pesticides, herbicides, antibiotics, anabolic hormones and other chemical and synthetic components. The processing should not involve the use of high temperatures or pressures, due to the possible reduction in the biological activity of its components under such conditions. The more preferred form of colostrum is a highly concentrated solid form rather than a liquid. Unfortunately, due to its short shelf life, it is necessary to use preservatives when stored in warm environmental conditions. However, this is associated with a lack of protection against loss of some active ingredients. In order to create an optimal form of the product for 
Table 3. Components of bovine colostrum (Bagwe et al., 2015; Godhia and Patel, 2013)

\begin{tabular}{|c|c|}
\hline Constituents & Cow colostrum \\
\hline \multicolumn{2}{|c|}{ Water-soluble vitamins, $\mu \mathrm{g} / \mathrm{mL}$} \\
\hline Niacin & 0.34 \\
\hline Thiamine & 0.9 \\
\hline Riboflavin & 4.5 \\
\hline Vitamin $B_{12}$ & 0.6 \\
\hline \multicolumn{2}{|c|}{ Fat-soluble vitamins, $\mu \mathrm{g} / \mathrm{g}$} \\
\hline Retinol & 4.9 \\
\hline Tocopherol & 2.9 \\
\hline Beta-caroten & 0.7 \\
\hline Cholecalciferol & 0.0305 \\
\hline \multicolumn{2}{|c|}{ Minerals, mg/kg } \\
\hline Calcium & 4716 \\
\hline Phosphorus & 4452 \\
\hline Magnesium & 733 \\
\hline Sodium & 1058 \\
\hline Potassium & 2845 \\
\hline Zinc & 38 \\
\hline Iron & 5.3 \\
\hline Copper & 0.3 \\
\hline Suplhur & 2595 \\
\hline Manganese & 0.1 \\
\hline \multicolumn{2}{|c|}{ Immunoglobulins, $\mathrm{mg} / \mathrm{mL}$} \\
\hline IgG1 & 35 \\
\hline $\operatorname{IgG} 2$ & 16 \\
\hline IgA & 1.7 \\
\hline $\operatorname{IgM}$ & 4.3 \\
\hline Lactoferrin & 0.8 \\
\hline \multicolumn{2}{|c|}{ Growth factors } \\
\hline EGF, $\mu \mathrm{g} / \mathrm{L}$ & $30-50$ \\
\hline TGF- $\alpha, \mu \mathrm{g} / \mathrm{L}$ & $2.2-7.2$ \\
\hline TGF- $\beta, \mathrm{mg} / \mathrm{L}$ & $1-2$ \\
\hline IGF, mg/L & 10 \\
\hline $\mathrm{GH}, \mathrm{ng} / \mathrm{L}$ & $<0.03$ \\
\hline
\end{tabular}

oral supply, fats, whey and lactose should be eliminated. The process of colostrum supplement production involves pasteurisation at low temperatures and indirect steam drying (Bagwe et al., 2015).
Synthetic colostrum is referred to as "false" colostrum. For the production of such colostrum, milk, egg yolks, cod liver oil and sugars are used. Such a formula is used to feed young animals when the mother cannot produce the right amount of colostrum. This serves only as a temporary replacement because it is low in antibodies. However, the synthetic colostrum which is produced for newborns uses precisely measured ingredients, such as carbohydrates, amino acids, vitamins and trace elements (Bagwe et al., 2015).

\section{COLOSTRUM BOVINUM AND SPORT BENEFITS}

Colostrum, thanks to its health properties, has been known for centuries. The data show that the constituents of bovine colostrum have a ten- or even a one thousand-fold more intense effect than human colostrum (Bagwe et al., 2015). The immunotropic properties of colostrum proteins have been subject to clinical trials and animal models. Based on the results, it is possible to demonstrate their usefulness in the prevention and treatment of autoimmune, neoplastic, immunodeficiency diseases, after chemotherapy or sepsis. The promising results of such research encouraged the use of colostrum in the pharmaceutical industry (Zimecki and Artym, 2005).

The first study on colostrum supplementation in sport was carried out by Mero et al. (1997) in 1997. The effect of colostrum on the concentration of immunoglobulins in the blood serum and on explosive muscle power was checked. Since then, researchers have been investigating the ability of colostrum to increase endurance and strength, improve anaerobic capacity, and determine the mechanisms responsible for the beneficial effect of colostrum on an athlete's performance. Of particular interest is the beneficial effect of colostrum on the functionality of the immune system (Shing et al., 2009).

\section{Immune system}

Colostrum provides newborns with immunoglobulins, maternal lymphocytes and cytokines, which contribute to the development of mucosal immunity. Unlike humans, in whom IgG transport takes place in the uterus, through the placenta, the transfer of immunoglobulins in calves is only possible through colostrum from mammary glands. The first few hours after 
calving are crucial for the development of passive immunity in calves, due to the increased ability to absorb macromolecules, which then gradually decreases and is completed about 24 hours after parturition. Insufficient supply of colostrum is associated with increased mortality in calves while, on the other hand, a high supply of it significantly increases the development of the gastrointestinal tract and the immune system (Shing et al., 2009).

The significant impact of colostrum supply on the development of the immune system of calves has led to the use of colostrum supplementation in humans to improve their immune functions. Intense physical activity suppresses immunity up to several hours after training, which is known as the "open window". Due to the large volume of highly intense exercise that endurance athletes undergo, they are at high risk of immunological disorders (Shing et al., 2009).

It is worth noting that the mechanisms of colostrum influence on humans are different than in the case of calves. There does not seem to be any passive transfer, or the possibility of survival of antibodies, in the digestive process. It seems more likely that bioactive compounds or their metabolites appear after ingestion, digestion and absorption processes, and they have a direct effect on the immune system. In fact, colostrum supplementation has been shown to enhance the immune system, both in in vitro leukocyte work and in vitro humoral responses. For this reason, there are further studies that test the possibilities of colostrum supply with respect to immunology (Shing et al., 2009).

In one of these studies, a 33\% increase in SIgA was already observed after two weeks of colostrum supplementation at a dose of $20 \mathrm{~g}$ per day (Mero et al., 2002). In a later study, the use of a 12 -week period of supplementation of a chocolate drink containing $12 \mathrm{~g}$ colostrum per day in a group of runners led to a $79 \%$ increase in resting SIgA (Crooks et al., 2006). The concentration of this immunoglobulin is a good indicator for predicting the risk of upper respiratory tract infections (URTI) in athletes. On the contrary, some of the studies found no significant difference in SIgA between supplemented and placebo groups (Crooks et al., 2010; Davison and Diment, 2010; Jones et al., 2014; 2015; Mero et al., 1997; Shing et al., 2007; 2013).

Brinkworth and Buckley (2003) showed a reduced frequency of upper respiratory tract diseases in physically active individuals receiving $60 \mathrm{~g}$ of colostrum per day for a period of eight weeks. Other studies have demonstrated the beneficial effect of colostrum supplementation on some markers of the immune system which are related to defense against infection in athletes. Davison and Diment (2010) reported a protective effect on the decline of lysozyme in saliva after four weeks of supplementation with $20 \mathrm{~g}$ of colostrum per day. Moreover, the positive effect of colostrum on some other immune markers, except for SIgA was revealed in another study (Shing et al., 2007). An investigation carried out by Crooks et al. (2010) on a group of swimmers using $25 \mathrm{~g}$ of colostrum per day for a period of 10 weeks showed a reduction in the reporting of health problems related to URTI. However, no measurable effect on the concentration of immunoglobulins was observed in this study. Similarly, Carol et al. (2011), using the same supplementation protocol, did not show any effect of the colostrum supply on the immune system during intense workouts. As a matter of fact, the markers used in this study have relatively little significance in the evaluation of the functioning and modulation of the immune system.

In the last 4 years, further research has been conducted on the impact of colostrum on exercise immunology. A study by Jones et al. (2014) tested the effect of a 12-week supplementation of $20 \mathrm{~g}$ of colostrum on URTI in physically active people. A reduction of the normal increase in the salivary bacterial load in winter demonstrated in this study indicates the immunomodulatory properties of colostrum. Another study involved four weeks of supplementation with $20 \mathrm{~g}$ of colostrum in recreationally active individuals (Jones et al., 2015). A beneficial effect on receptors modulating the stimulation of neutrophil oxidative burst was found during this study. A few years later, the same researcher used the same supplementation protocol and showed that colostrum blunts the prolonged exercise-induced decrease in in vivo immune responsiveness to a novel antigen (Jones et al., 2017). In a later study, Kotsis et al. (2018) demonstrated that the administration of $3.2 \mathrm{~g}$ of colostrum for six weeks led to a reduction in exercise-induced muscle damage and markers of inflammation.

Based on the presented results, it can be concluded that colostrum supplementation may have a beneficial effect on the immune system of athletes. However, it is necessary to conduct standardization tests on the dose 
and period of effective supplementation and to identify markers of immune system adequate for the evaluation of its response in the case of such stimulation.

\section{Digestive system}

The differentiation and maturation of digestive tract cells is observed in calves after colostrum supply. Growth factors present in the colostrum play an important role in the growth, proliferation and repair of the gastrointestinal tract. The potential of using colostrum in humans to improve the digestive system has led to the commencement of scientific research in this direction. The beneficial effects of colostrum consumption were demonstrated in colitis, histological patterns, intensity of symptoms and duration of intestinal problems. In healthy people, colostrum may reduce excessive intestinal permeability or inflammation caused by the chronic use of non-steroidal anti-inflammatory drugs and antibiotics, whereas it does not affect the absorption of nutrients. It turns out that a supply of colostrum may only be beneficial in cases of inflammation and disturbances of the gastric mucosa (Shing et al., 2009).

An increase in intestinal barrier permeability may impair the performance of athletes as a result of bacterial endotoxin translocation, nausea and diarrhea. Colostrum supplementation may show benefits during intense periods of training that may affect gut permeability, especially at high environmental temperatures (Shing et al., 2009).

The first test to check the effects of colostrum on the intestinal barrier in athletes was carried out by Buckley et al. in 2009. Unexpectedly, it showed that 8 -week supplementation of $60 \mathrm{~g}$ of colostrum per day increased the permeability of the intestinal barrier, in contrast to the earlier results of studies in rats. Another study in this area used a supply of $20 \mathrm{~g}$ of colostrum for 14 days and tested the ratio of lactulose to rhamnose in the urine - a marker of permeability (Marchbank et al., 2011). Colostrum has been demonstrated to be effective in maintaining the stability of the intestinal membrane by reducing apoptosis and intercellular permeability. In another report, using a dosage of $1.7 \mathrm{~g}$ of colostrum per $\mathrm{kg}$ of body weight for seven days, the concentration of circulating pro- and antiinflammatory cytokines was not modulated by supplementation. Accordingly, colostrum was found to show no observable benefits on the physiology or exercise performance of physically active individuals (Morrison et al., 2014). Davison et al. (2016) studied the use of zinc carnosine $(75 \mathrm{mg})$ taken with and without colostrum $(20 \mathrm{~g})$ on increased intestinal permeability induced by severe physical exercise. In both cases, an increase in epithelial resistance and a tight structure of the junctions was demonstrated. A Polish study conducted on a group of MMA players by Hałasa et al. (2017) used $500 \mathrm{mg}$ of colostrum for 20 days. The concentration of zonulin in the faeces and the ratio of lactulose to mannitol were checked. In this instance, colostrum supplementation was shown to be effective in reducing intestinal permeability. In a later study by March et al. (2018), $20 \mathrm{~g}$ of colostrum was applied for a period of 14 days. This was the first study that showed that colostrum may be effective in reducing intestinal pain caused by exercise at high temperatures; however, there was no effect on the circulating concentration of bacterial DNA.

It can be assumed that colostrum supplementation could have a beneficial effect on the intestinal barrier in athletes under some training circumstances, but further research is needed in this direction, especially regarding the mechanism of its action.

\section{Neuroendocrine system}

Colostrum affects the maturation of the hypothalamicpituitary axis (HPA), and thus the functioning of the neuroendocrine system of calves, through the direct transport of colostral proteins to the cerebrospinal fluid. Disorders of the HPA, which can be induced by cytokines, are associated with mood disorders and fatigue. It turns out that colostrum contains hormones, such as growth factors, gonadotrophin releasing hormone, luteinizing hormone, glucocorticoids or testosterone, that affect HPA and the production of sex hormones. Unfortunately, it is not entirely clear what the functions of the hormones present in colostrum are. However, it is believed that they play an important role in the development of the digestive, immune or neuroendocrine systems. For now, it has not been established whether the components of colostrum are able to penetrate the blood-brain barrier (Shing et al., 2009).

Intensive workouts are associated with mood disorders that can be triggered by changes in hypothalamic neuropeptides. However, it has been shown that supplementation of $20 \mathrm{~g}$ of colostrum for eight weeks 
is associated with a decrease in fatigue and increased vigor for training in healthy men (Shing et al., 2009).

A pilot study by Shing et al. (2013) tested the effects of colostrum on the concentration of hormones and IgA in the saliva of competitive cyclists. The results showed that testosterone levels were maintained and autonomic activity in the days after cycling events was modulated. There is a need for further research in the field of recovery of the neuroendocrine system in athletes through colostrum supply.

Based on the sparse data presented, it can be presumed that colostrum supplementation has the potential to be effective in mood improvement, which may reflect its influence on neuroendocrine system homeostasis. However, it is necessary to conduct further research on athletes to better understand this issue and provide more convincing evidence.

\section{Exercise performance}

Colostrum supplementation is also popular among athletes for increasing lean body mass and improving performance. The first study in this area was conducted in 1997 (Mero et al., 1997), within which the impact of colostrum on explosive muscle power was studied. Subsequent research concerned endurance, strength, anaerobic capacity and the mechanisms responsible for improving athletic performance as a result of colostrum supply (Shing et al., 2009).

Based on the results of a few studies (Buckley et al., 2002; Coombes et al., 2002; Shing et al., 2006), it can be concluded that the supply of colostrum may improve recovery after repeated exercise and the efficiency of time-trial tasks after prolonged submaximal effort, in addition to maintaining exercise performance after high-intensity training.

In a study by Buckley et al. (2002), a dose of $60 \mathrm{~g}$ of colostrum showed no significant differences in physical performance after four weeks. Nevertheless, after eight weeks of supplementation, runners were able to overcome a greater distance than at the beginning of the study. Interestingly, no increase in maximum oxygen uptake was noted. The mechanisms responsible for a significant improvement in runners' performance have not been explained. In the Coombes et al. (2002) study, changes in physical performance were checked in response to different doses of colostrum (20 vs $60 \mathrm{~g}$ for eight weeks). Significant, but not dose-dependent improvements in cycling performance were demonstrated, suggesting that there may be an upper limit of colostrum supply, above which no major benefits are observed. Other studies revealed that colostrum supplementation at doses of $60 \mathrm{~g}$ for eight weeks gave mixed results in improving vertical jump height and peak cycle power (Buckley et al., 2002; Hofman et al., 2002). One of the studies by Shing et al. (2006) showed that consumption of colostrum may help to maintain the oxygen threshold and improve exercise economy during high-intensity training. Colostrum supplementation has been shown to improve endurance performance, though these changes may be independent of increases in IGF-I, as was suggested in previous studies (Shing et al., 2009). The other proposed mechanism is based on studies in calves, in which it was demonstrated that colostrum increases blood glucose and gluconeogenesis. However, this requires confirmation in research on physically active people (Shing et al., 2009). Duff et al. (2014) used $60 \mathrm{~g}$ of colostrum in an older population for eight weeks and noticed improved muscle strength indicating that the advantage of this supplement might not be limited by age.

Due to the positive effect of colostrum supply on the increase of circulating proteins and stimulation of skeletal muscle growth in calves, it is possible to gain benefits for anaerobic performance. Few data from scientific studies (Antonio et al., 2001; Brinkworth et al., 2002) showed that colostrum may induce an increase in the protein content of muscles and blood buffer capacity. A significantly higher lean tissue mass was reported in the supplemented group of active individuals (Antonio et al., 2001). On the other hand, Duff et al. (2014) did not find any differences in the body composition of older adults during resistance training. However, in another study on female rowers (Brinkworth and Buckley, 2004), there were significant changes in lean tissue mass but no significant differences in the concentration of resting haemoglobin and bicarbonates in the blood or the buffering capacity of blood between the groups administered with colostrum and a placebo. Nevertheless, it was concluded that the previously observed increase in buffering capacity was the result of increased muscle buffering capacity. Further research in this direction is necessary.

In conclusion, colostrum supplementation seems to be beneficial for endurance, especially during intense 
training. However, there is no conclusive evidence for colostrum's effect on the strength or body composition of athletes. In the case of anaerobic capacity, there are currently insufficient studies confirming the beneficial effects of colostrum supplementation.

\section{IGF-I}

In accordance with the guidelines of the AIS Sports Supplement Framework 2019 (Australian Sports Commission, 2019), colostrum belongs to group D (supplements banned or at high risk of contamination with substances that could lead to a positive doping test). It is not forbidden but nor is it recommended by the World Anti-Doping Agency due to the inclusion of growth factors within its composition. Nevertheless, despite the fact that colostrum contains IGF-I, there are only two studies (Mero et al., 1997; 2002) that reported significant increases in IGF-I serum levels after colostrum supplementation (respectively $25 / 125$ $\mathrm{ml}$ of colostrum supplement and $20 \mathrm{~g}$ of colostrum). It is worth noting that IGF-I is degraded in the gastrointestinal tract, but it is possible that some factors present in colostrum may prevent its breakdown and help improve its absorption. The normal serum level of IGF-I for young adults is $14-48 \mathrm{nmol} / 1$ (Watson et al., 2017), for adults $18-50$ years old it is $5-58 \mathrm{nmol} / 1$
(Mayo Clinic Labs, n.d.), while the increase reported in previous studies was approximately $5 \mathrm{nmol} / 1$ (at the administrated amount of $74 \mu \mathrm{g} /$ day of IGF-I in 20 $\mathrm{g}$ of colostrum). It was reported that the serum concentration of IGF-I after colostrum supplementation did not exceed the upper limit of the reference level (Mero et al., 1997; 2002). Even if, at this dose, 65\% of IGF-I was absorbed, the expected rise would only be by $1.05 \mathrm{nmol} / 1$. It is suggested that a higher increase in serum IGF-I concentration was induced by other factors after the supplementation, like increased endogenous production (Shing et al., 2009; Watson et al., 2017).

\section{CONCLUSIONS}

Colostrum Bovinum contains a number of proteins, immune components and hormones that are similar to the bioactive compounds present in human colostrum. The influence of colostrum supply on the development of calves is fairly well understood, nevertheless there is not enough data to explain the mechanisms of colostrum action in humans. Despite the widespread use of colostrum supplementation among athletes, the conclusions drawn from scientific publications investigating the effects of colostrum on exercise performance are ambiguous (Table 4). Currently, it is stated that the

Table 4. Summary of possible colostrum outcomes in sport

\begin{tabular}{|c|c|c|}
\hline & Outcomes & References \\
\hline Immune system & $\begin{array}{l}\text { immunoglobulins: possible spikes in saliva and serum IgA, } \\
\text { no effects on serum IgG, IgM and IgE } \\
\text { sickness: a reduction in the frequency of sickness } \\
\text { and the incidence of URTI }\end{array}$ & $\begin{array}{l}\text { Brinkworth and Buckley, 2003; Carol } \\
\text { et al., 2011; Crooks et al., 2006; } \\
\text { 2010; Mero et al., 2002; Shing et al., } \\
\text { 2007; } 2013\end{array}$ \\
\hline Digestive system & $\begin{array}{l}\text { intestinal permeability: possible beneficial effects on intestinal } \\
\text { permeability after exercise }\end{array}$ & $\begin{array}{l}\text { Buckley et al., 2009; Marchbank } \\
\text { et al., } 2011\end{array}$ \\
\hline Neuroendocrine system & $\begin{array}{l}\text { possible decrease in fatigue, increased mood and maintained } \\
\text { neuroendocrine homeostasis }\end{array}$ & Shing et al., 2009; 2013 \\
\hline Exercise performance & $\begin{array}{l}\text { body weight: no significant effects on body composition } \\
\text { or possible lean mass gain } \\
\text { aerobic performance: possible ergogenic benefits in the occur- } \\
\text { rence of high endurance stressors } \\
\text { anaerobic performance: no benefits in improving anaerobic } \\
\text { performance } \\
\text { muscle power output: no effects }\end{array}$ & $\begin{array}{l}\text { Antonio et al., 2001; Brinkworth } \\
\text { et al., 2002; 2004; Buckley et al., } \\
\text { 2002; 2003; 2009; Carol et al., 2011; } \\
\text { Coombes et al., 2002; Crooks et al., } \\
\text { 2006; 2010; Hofman et al., 2002; } \\
\text { Mero et al., 1997; Shing et al., } 2006\end{array}$ \\
\hline IGF-I & $\begin{array}{l}\text { the same IGF-I increase as with whey, fully digested } \\
\text { in the gastrointestinal tract }\end{array}$ & $\begin{array}{l}\text { Buckley et al., 2002; 2003; Coombes } \\
\text { et al., 2002; Mero et al., 1997; } 2002\end{array}$ \\
\hline
\end{tabular}


supply of colostrum has benefits for the immune system, intestinal barrier and mood. A positive effect on endurance and anaerobic efficiency is not fully confirmed but possible. Due to conflicting results on the impact of colostrum on the health and physical performance of athletes, it is necessary to carry out further properly prepared clinical trials using standardized doses of colostrum and sufficiently long supplementation periods.

\section{REFERENCES}

Antonio, J., Sanders, M. S., Van Gammeren, D. (2001). The effects of bovine colostrum supplementation on body composition and exercise performance in active men and women. Nutrition, 17(3), 243-247.

Australian Sports Commission (2019). The AIS Sports Supplement Framework. Retrieved March 13, 2019, from: https://www.sportaus.gov.au/ais/nutrition/supplements

Bagwe, S., Tharappel, L. J. P., Kaur, G., Buttar, H. S. (2015). Bovine colostrum: an emerging nutraceutical. J. Compl. Integr. Med., 12(3), 175-185. https://doi.org/10.1515/ jcim-2014-0039

Brinkworth, G. D., Buckley, J. D. (2003). Concentrated bovine colostrum protein supplementation reduces the incidence of self-reported symptoms of upper respiratory tract infection in adult males. Eur. J. Nutr., 42(4), 228-232. https://doi.org/10.1007/s00394-003-0410-x

Brinkworth, G. D., Buckley, J. D. (2004). Bovine colostrum supplementation does not affect plasma buffer capacity or haemoglobin content in elite female rowers. Eur. J. Appl. Physiol., 91(2-3), 353-356. https://doi. org/10.1007/s00421-003-1023-z

Brinkworth, Grant D., Buckley, J. D., Slavotinek, J. P., Kurmis, A. P. (2004). Effect of bovine colostrum supplementation on the composition of resistance trained and untrained limbs in healthy young men. Eur. J. Appl. Physiol., 91(1), 53-60. https://doi.org/10.1007/s00421003-0944-x

Brinkworth, Grant David, Buckley, J. D., Bourdon, P. C., Gulbin, J. P., David, A. (2002). Oral bovine colostrum supplementation enhances buffer capacity but not rowing performance in elite female rowers. Int. J. Sport Nutr. Exer. Metab., 12(3), 349-365.

Buckley, J., Abbott, M., Brinkworth, G., Whyte, P. (2002). Bovine colostrum supplementation during endurance running training improves recovery, but not performance. J. Sci. Med. Sport, 5(2), 65-79.
Buckley, J., Butler, R., Southcott, E., Brinkworth, G. (2009). Bovine colostrum supplementation during running training increases intestinal permeability. Nutrients, 1(2), 224-234. https://doi.org/10.3390/nu1020224

Buckley, J. D., Brinkworth, G. D., Abbott, M. J. (2003). Effect of bovine colostrum on anaerobic exercise performance and plasma insulin-like growth factor I. J. Sports Sci., 21(7), 577-588. https://doi.org/10.1080/0264041031000 101935

Carol, A., Witkamp, R. F., Wichers, H. J., Mensink, M. (2011). Bovine colostrum supplementation's lack of effect on immune variables during short-term intense exercise in well-trained athletes. Int. J. Sport Nutr. Exe., 21(2), 135-145.

Coombes, J. S., Conacher, M., Austen, S. K., Marshall, P. A. (2002). Dose effects of oral bovine colostrum on physical work capacity in cyclists. Med. Sci. Sports Exe., 34(7), 1184-1188.

Crooks, C., Cross, M. L., Wall, C., Ali, A. (2010). Effect of bovine colostrum supplementation on respiratory tract mucosal defenses in swimmers. Int. J. Sport Nutr. Exe., 20(3), 224-235.

Crooks, C., Wall, C. R., Cross, M. L., Rutherfurd-Markwick, K. J. (2006). The effect of bovine colostrum supplementation on salivary IgA in distance runners. Int. J. Sport Nutr. Exe., 16(1), 47-64.

Davison, G. (2012). Bovine colostrum and immune function after exercise. Med. Sport Sci., 59, 62-69.

Davison, G., Diment, B. C. (2010). Bovine colostrum supplementation attenuates the decrease of salivary lysozyme and enhances the recovery of neutrophil function after prolonged exercise. Brit. J. Nutr., 103(10), 1425-1432. https://doi.org/10.1017/S0007114509993503

Davison, G., Marchbank, T., March, D. S., Thatcher, R., Playford, R. J. (2016). Zinc carnosine works with bovine colostrum in truncating heavy exercise-induced increase in gut permeability in healthy volunteers. Am. J. Clin. Nutr., 104(2), 526-536. https://doi.org/10.3945/ ajcn.116.134403

Duff, W. R. D., Chilibeck, P. D., Rooke, J. J., Kaviani, M., Krentz, J. R., Haines, D. M. (2014). The effect of bovine colostrum supplementation in older adults during resistance training. Int. J. Sport Nutr. Exe., 24(3), 276-285. https://doi.org/10.1123/ijsnem.2013-0182

Francis, G. L., Upton, F. M., Ballard, F. J., McNeil, K. A., Wallace, J. C. (1988). Insulin-like growth factors 1 and 2 in bovine colostrum. Sequences and biological activities compared with those of a potent truncated form. Biochem. J., 251(1), 95-103. 
Godhia, M. L., Patel, N. (2013). Colostrum - its composition, benefits as a nutraceutical. A review. Curr. Res. Nutr. Food Sci., 1(1), 37-47. http://dx.doi.org/10.12944/ CRNFSJ.1.1.04

Hałasa, M., Maciejewska, D., Baśkiewicz-Hałasa, M., Machaliński, B., Safranow, K., Stachowska, E. (2017). Oral supplementation with bovine colostrum decreases intestinal permeability and stool concentrations of zonulin in athletes. Nutrients, 9(4), 370. https://doi. org/10.3390/nu9040370

Hofman, Z., Smeets, R., Verlaan, G., Lugt, R., Verstappen, P. A. (2002). The effect of bovine colostrum supplementation on exercise performance in elite field hockey players. Int. J. Sport Nutr. Exe., 12(4), 461-469.

Jones, A. W., Cameron, S. J. S., Thatcher, R., Beecroft, M. S., Mur, L. A. J., Davison, G. (2014). Effects of bovine colostrum supplementation on upper respiratory illness in active males. Brain Behav. Immun., 39, 194-203. https://doi.org/10.1016/j.bbi.2013.10.032

Jones, A. W., March, D. S., Thatcher, R., Diment, B., Walsh, N. P., Davison, G. (2017). The effects of bovine colostrum supplementation on in vivo immunity following prolonged exercise: a randomised controlled trial. Eur. J. Nutr., 58, 1, 335-344. https://doi.org/10.1007/s00394017-1597-6

Jones, A. W., Thatcher, R., March, D. S., Davison, G. (2015). Influence of 4 weeks of bovine colostrum supplementation on neutrophil and mucosal immune responses to prolonged cycling. Scand. J. Med. Sci. Sports, 25(6), 788-796. https://doi.org/10.1111/sms.12433

Kotsis, Y., Mikellidi, A., Aresti, C., Persia, E., Sotiropoulos, A., Panagiotakos, D. B., ..., Nomikos, T. (2018). A lowdose, 6-week bovine colostrum supplementation maintains performance and attenuates inflammatory indices following a Loughborough Intermittent Shuttle Test in soccer players. Eur. J. Nutr., 57(3), 1181-1195. https:// doi.org/10.1007/s00394-017-1401-7

March, D. S., Jones, A. W., Thatcher, R., Davison, G. (2018). The effect of bovine colostrum supplementation on intestinal injury and circulating intestinal bacterial DNA following exercise in the heat. Eur. J. Nutr., https://doi. org/10.1007/s00394-018-1670-9

Marchbank, T., Davison, G., Oakes, J. R., Ghatei, M. A., Patterson, M., Moyer, M. P., Playford, R. J. (2011). The nutriceutical bovine colostrum truncates the increase in gut permeability caused by heavy exercise in athletes. Am. J. Physiol. Gastroint. Liver Physiol., 300(3), G477484. https://doi.org/10.1152/ajpgi.00281.2010

Mayo Clinic Labs. (n.d.). IGFGP - Clinical: Insulin-Like Growth Factor 1 (IGF1), LC-MS and Insulin-Like
Growth Factor-Binding Protein 3 (IGFBP3) Growth Panel. Retrieved March 22, 2019, from: https://www. mayocliniclabs.com/test-catalog/Clinical\%2Band\%2BI nterpretive $/ 36365$

Mero, A., Kähkönen, J., Nykänen, T., Parviainen, T., Jokinen, I., Takala, T., ..., Leppäluoto, J. (2002). IGF-I, IgA, and $\mathrm{IgG}$ responses to bovine colostrum supplementation during training. J. Appl. Physiol., 93(2), 732-739. https:// doi.org/10.1152/japplphysiol.00002.2002

Mero, A., Miikkulainen, H., Riski, J., Pakkanen, R., Aalto, J., Takala, T. (1997). Effects of bovine colostrum supplementation on serum IGF-I, IgG, hormone, and saliva IgA during training. J. Appl. Physiol., 83(4), 1144-1151. https://doi.org/10.1152/jappl.1997.83.4.1144

Morrison, S. A., Cheung, S. S., Cotter, J. D. (2014). Bovine colostrum, training status, and gastrointestinal permeability during exercise in the heat: a placebo-controlled double-blind study. Appl. Physiol. Nutr. Metab., 39(9), 1070-1082. https://doi.org/10.1139/apnm-2013-0583

Rak, K., Bronkowska, M. (2014). Immunologiczne znaczenie siary [Immunological role of colostrum]. Hyg. Publ. Health, 49(2), 249-254 [in Polish].

Shing, C. M., Hunter, D., Stevenson, L. (2009). Bovine colostrum supplementation and exercise performance: potential mechanisms. Sports Med., 39(12), 1033-1054. https://doi.org/10.2165/11317860-000000000-00000

Shing, C. M., Jenkins, D. G., Stevenson, L., Coombes, J. S. (2006). The influence of bovine colostrum supplementation on exercise performance in highly trained cyclists. Brit. J. Sports Med., 40(9), 797-801. https://doi. org/10.1136/bjsm.2006.027946

Shing, C. M., Peake, J. M., Suzuki, K., Jenkins, D. G., Coombes, J. S. (2013). A pilot study: bovine colostrum supplementation and hormonal and autonomic responses to competitive cycling. J. Sports Med. Phys. Fit., 53(5), 490-501.

Shing, C. M., Peake, J., Suzuki, K., Okutsu, M., Pereira, R., Stevenson, L., ..., Coombes, J. (2007). Effects of bovine colostrum supplementation on immune variables in highly trained cyclists. J. Appl. Phys., 102(3), 11131122. https://doi.org/10.1152/japplphysiol.00553.2006

Watson, R. R., Collier, R. J., Preedy, V. R. (2017). Nutrients in dairy and their implications for health and disease. Academic Press.

Zimecki, M., Artym, J. (2005). Właściwości terapeutyczne białek i peptydów z siary i mleka. Post. Hig. Med. Dośw., 59(0). Retrieved from: https://phmd.pl/resources/html/ article/details?id $=6568$ 